\title{
Schizandrin $B$ attenuates hypoxia/reoxygenation injury in H9c2 cells by activating the AMPK/Nrf2 signaling pathway
}

\author{
BO ZHAO ${ }^{1}$, GUANG-PING LI ${ }^{1,2}$, JIAN-JUN PENG ${ }^{1}$, LI-HUI REN ${ }^{1}$, \\ LI-CHENG LEI ${ }^{1}$, HUI-MING YE ${ }^{1}$, ZUO-YAN WANG ${ }^{1}$ and SHENG ZHAO ${ }^{1}$ \\ ${ }^{1}$ Department of Cardiology, Beijing Shijitan Hospital, Capital Medical University, Beijing 100038; \\ ${ }^{2}$ Department of Cardiology, Tianjin Key Laboratory of Ionic-Molecular Function of Cardiovascular Disease, \\ Tianjin Institute of Cardiology, The Second Hospital of Tianjin Medical University, Tianjin 300211, P.R. China
}

Received December 1, 2019; Accepted June 10, 2020

DOI: 10.3892/etm.2021.9651

\begin{abstract}
Schizandrin B exhibits prominent antioxidant and anti-inflammatory effects, and plays an important role in ameliorating myocardial ischemia/reperfusion injury. However, the underlying protective mechanisms remain to be elucidated. The aim of the present study was to explore the cardioprotective effects of schizandrin $B$ against hypoxia/reoxygenation (H/R)-induced H9c2 cell injury, focusing on the role of the adenosine monophosphate-activated protein kinase (AMPK)/nuclear factor erythroid 2-related factor 2 (Nrf2) pathway in this process. The results showed that schizandrin $B$ attenuated the $\mathrm{H} / \mathrm{R}$-induced decrease in cell viability and the increase in lactate dehydrogenase release, as well as the apoptosis rate in H9c2 cells. Schizandrin B also mitigated H/R-induced oxidative stress, as illustrated by the decrease in intracellular reactive oxygen species generation, malondialdehyde content and NADPH oxidase 2 expression, and the increase in antioxidant enzyme superoxide dismutase and glutathione peroxidase activities. In addition, schizandrin $B$ reversed the H/R-induced upregulation of pro-inflammatory cytokines [interleukin (IL)-1 $\beta$ (IL-1 $\beta$ ) tumor necrosis factor- $\alpha$, IL-6 and IL-8] and the downregulation of anti-inflammatory cytokines (transforming growth factor- $\beta$ and IL-10) in the culture supernatant. Notably, schizandrin B increased the expression of $\mathrm{Nrf} 2, \mathrm{NAD}(\mathrm{P}) \mathrm{H}$ : Quinone oxidoreductase (NQO-1) and heme oxygenase-1 (HO-1) in H/R-treated H9c2 cells, activating the Nrf2 signaling pathway. The cardioprotection of schizandrin B against H/R injury was inhibited by Nrf2 knockdown induced byNrf-2-specific small interfering RNA
\end{abstract}

Correspondence to: Dr Guang-Ping Li, Department of Cardiology, Beijing Shijitan Hospital, Capital Medical University, 10 Tieyi Road, Haidian, Beijing 100038, P.R. China

E-mail: ligpusc0102@qq.com

Key words: schizandrin B, myocardial ischemia/reperfusion injury, oxidative stress, inflammation, adenosine monophosphate-activated protein kinase/nuclear factor erythroid 2-related factor 2signaling pathway
(siRNA; si-Nrf2) transfection. Furthermore, schizandrin B enhanced phosphorylated (p)-AMPK expression, while AMPK knockdown induced by AMPK-specific siRNA(si-AMPK) transfection remarkably eliminated schizandrin B-induced cardioprotection and reduced Nrf2 expression in H/R-treated H9c2 cells. Taken together, these results suggested that schizandrin B exerts cardioprotection on H/R injury in H9c2 cells due to its antioxidant and anti-inflammatory activities via activation of the AMPK/Nrf2 pathway.

\section{Introduction}

Myocardial ischemia-reperfusion injury (MIRI) is one of the major diseases threatening human health with a high morbidity and mortality rate worldwide $(1,2)$. A number of pathological processes and mediators, including cell apoptosis, oxidative stress injury, intracellular calcium overload and inflammatory response activation have been proposed to be crucial in ischemia-reperfusion (I/R)-related myocardial cell injury $(3,4)$. Among them, oxidative stress and inflammation are considered important mechanisms implicated in the pathogenesis of MIRI $(5,6)$. During the phase of MIRI, oxidative stress occurs when there are imbalances between reactive oxygen species (ROS) generation and antioxidant defense systems, leading to the activation of signal transduction cascades and the production of various inflammatory mediators, which results in damage of the viable tissue surrounding the infarct and accelerated cell death programs (7-9). Despite the advances in the understanding of MIRI mechanisms (1), novel effective strategies for MIRI remain to be explored.

Numerous traditional Chinese herbs have been proven to exert cardioprotective effects, and their role in ameliorating MIRI has been investigated (10-12). Schizandrin B, the most abundant active dibenzocyclooctadiene derivative isolated from a traditional Chinese herb [Schisandra chinensis (Turcz) Baill], possesses diverse pharmacological activities such as anti-apoptosis, antioxidative, anti-inflammatory and cardioprotective properties (13-15). A previous in vivo study demonstrated that schizandrin B might protect myocardial tissue from I/R injury via the phosphoinositide 3-kinase/Akt signaling pathway in rats (15). In addition, a previous study demonstrated that schizandrin $\mathrm{B}$ has a high antioxidative 
activity and protects against MIRI (16). However, the potential mechanism of schizandrin B-induced cardioprotection against MIRI remains elusive.

Adenosine monophosphate-activated protein kinase (AMPK) is a major regulator of cellular homeostasis, and its activation can reduce oxidative stress injury and the inflammatory response $(17,18)$. Increasing evidence indicated that AMPK regulates a variety of biological processes and is a highly effective therapeutic target for protecting against MIRI (19-21). Nuclear factor erythroid 2-related factor 2 (Nrf2) is essential for the transcription and expression of key antioxidant enzymes, including heme oxygenase-1 (HO-1) and $\mathrm{NAD}(\mathrm{P}) \mathrm{H}$ dehydrogenase quinone 1 (NQO-1) $(22,23)$. Under normal conditions, the transcriptional activity of the Nrf2 protein is inhibited by the negative regulator Kelch-like ECH-associated protein 1 (Keap1); however, upon excess oxidative stress, Nrf2 translocates to the nucleus, where it binds to the antioxidant responsive element $(24,25)$. Numerous studies revealed that $\mathrm{Nrf} 2$ served as an important downstream target of AMPK signaling by increasing resistance to oxidative damage and inflammatory reaction $(11,26)$. The AMPK/Nrf2 signaling pathway plays an important role in cellular defense against oxidative stress and inflammatory injury by activating antioxidant cascades $(27,28)$. Notably, previous studies confirmed that the mechanisms of these antioxidative and anti-inflammatory activities of schizandrin B are mediated, at least in part, via activation of Nrf2 and Nrf2-driven antioxidant responses $(29,30)$. However, the potential roles of the AMPK/Nrf2 signaling pathway in the cardioprotection of schizandrin B in MIRI remain to be elucidated.

Hence, in the present study, a cell model of MIRI was established to investigate whether schizandrin B attenuates oxidative stress and inflammatory response via the AMPK/Nrf2 signaling pathway, resulting in cardioprotection against MIRI, and provide a theoretical foundation for its clinical application.

\section{Materials and methods}

Materials and reagents. Dulbecco's modified Eagle's medium (DMEM; cat. no. 11965118) and fetal bovine serum (FBS; cat. no. 16140071) were supplied by Gibco; Thermo Fisher Scientific, Inc. Annexin V/fluorescein isothiocyanate (FITC) apoptosis detection kit (cat. no. 556547) was purchased from BD Biosciences. Cell counting kit-8 (CCK-8; cat. no. C0038), JC-1 mitochondrial membrane potential detection kit (cat. no. C2006) and lactate dehydrogenase (LDH) cytotoxicity assay kit (cat. no. C0016) were purchased from Beyotime Institute of Biotechnology. 2', 7'-dichlorofluorescein acetyl acetate (DCFH-DA; cat. no. D6883) kit was supplied by Sigma-Aldrich; Merck KGaA. Rabbit polyclonal antibodies against B-cell lymphoma 2 (Bcl-2; cat. no. ab32124) and Bcl-2associated X protein (Bax; cat. no. ab32503) were purchased from Abcam. Rabbit monoclonal antibody against NAPDH oxidase 2 (NOX2; cat. no. ALX-350-100-C050) was purchased from Enzo Life Sciences, Inc. Rabbit monoclonal antibodies against histone H3 (cat. no. 7631), Keap 1 (cat. no. 8047), AMPKa (cat. no. 5832), phospho (p)-AMPKa (Ser485; cat. no. 2537), Nrf2 (cat. no. 12721) and GAPDH (cat. no. 5174) were obtained from Cell Signaling Technology, Inc.
Cell culture and hypoxia/reoxygenation $(H / R)$ injury model establishment. The H9c2 cardiomyocyte cell line (rat embryonic cardiomyoblasts) was purchased from Shanghai Institutes for Biological Sciences, and was maintained in DMEM supplemented with $10 \%(\mathrm{v} / \mathrm{v})$ FBS and $1 \%(\mathrm{v} / \mathrm{v})$ penicillinstreptomycin solution in a humidified atmosphere containing $95 \%$ air and $5 \% \mathrm{CO}_{2}$ at $37^{\circ} \mathrm{C}$. The medium was replaced every 2 days. To induce $\mathrm{H} / \mathrm{R}$ injury, $\mathrm{H} 9 \mathrm{c} 2$ cells were cultured with serum-free medium in an anaerobic chamber containing $1 \%$ $\mathrm{O}_{2}, 5 \% \mathrm{CO}_{2}$ and $94 \% \mathrm{~N}_{2}$ at $37^{\circ} \mathrm{C}$ for $6 \mathrm{~h}$ to establish hypoxia. Subsequently, the cells were cultured with normoxic medium in the presence of $95 \%$ air and $5 \% \mathrm{CO}_{2}$ at $37^{\circ} \mathrm{C}$ for $12 \mathrm{~h}$ in a humidified atmosphere to establish reoxygenation (31).

Cell viability assay. Cell viability was determined using a CCK-8 assay kit according to the manufacturer's instructions. Briefly, H9c2 cells were seeded in a 96-well plate at a density of $3 \times 10^{4}$ cells/well. After treatment as described above, CCK- 8 solution $(10 \mu \mathrm{l})$ was added to each well and further incubated at $37^{\circ} \mathrm{C}$ for $3 \mathrm{~h}$. Subsequently, the optical density was measured at a wavelength of $450 \mathrm{~nm}$ using a PerkinElmer microplate reader (PerkinElmer, Inc.).

Small interfering RNA (siRNA) transfection. H9c2 cells were transfected with Nrf2-specific siRNA (si-Nrf2; $100 \mathrm{nM}$; cat. no. sc-37030), AMPK-specific siRNA (si-AMPK; $100 \mathrm{nM}$; cat. no. sc-29673) or scrambled siRNA (100 nM; cat. no. sc-37007) as the negative control (Santa Cruz Biotechnology, Inc.) using Lipofectamine 2000 (Invitrogen; Thermo Fisher Scientific, Inc.) according to the manufacturer's protocols. The sequences of the siRNA were as follows: Nrf2 siRNA sense, 5'-GAGGAUGGGAAACCUUACUTT-3' and antisense, 5'-AGUAAGGUUUCCCAUCCUCTT-3'; AMPK siRNA sense, 5'-CCCAUAUUAUUUGCGUGUADTDT-3' and antisense, 5'-UACACGCCAAAUAAUAUGGGCTCT-3' and scramble siRNA sense, 5'-UUCUCCGAACGUGUCACG UTT-3' and antisense, 5'-ACGUGACACGUUCGGAGAATT -3 '. Briefly, cells were plated in six-well plates prior to transfection. After growing to $60-70 \%$ confluence, siRNA (100 nM) was prepared in Opti-MEM ${ }^{\mathrm{TM}} \mathrm{I}(100 \mathrm{ml}$; Invitrogen; Thermo Fisher Scientific, Inc.; cat. no. 51985091) and then added to Opti-MEM $^{\mathrm{TM}}$ I $(100 \mathrm{ml})$ containing Lipofectamine ${ }^{\mathrm{TM}}$ Stem Transfection Reagent $(14 \mathrm{ml})$, and the mixture was incubated for $10 \mathrm{~min}$ at room temperature. The mixture was then added to the cells and incubated for $48 \mathrm{~h}$ prior to experimentation. Knockdown efficacy was determined by reverse transcriptionquantitative PCR (RT-qPCR).

RNA isolation and RT-qPCR assay. Total RNA from cells of the control, H/R, Sch B + H/R + si-Scram and Sch B + H/R + si-Nrf2 groups was isolated using TRIzol $^{\circledR}$ reagent (Invitrogen; Thermo Fisher Scientific, Inc.) following the manufacturer's protocol. RT of RNA ( $1 \mu \mathrm{g})$ to cDNA was performed using the M-MLV Reverse Transcriptase system (Promega Corporation) according to the manufacturer's instructions. The mRNA levels of AMPK and Nrf2 were detected by RT-qPCR using the SYBR Green PCR master mix (Applied Biosystems; Thermo Fisher Scientific, Inc.) on an ABI PRISM 7900 Sequence Detection system (Applied Biosystems; Thermo Fisher Scientific, Inc.). The following primer pairs were used 
for the qPCR: Nrf2 forward, 5'-ACTGTCCCCAGCCCAGAG GC-3'and reverse, 5'-CCAGGCGGTGGGTCTCCGTA-3'; HO-1 forward, 5'-GCTGGTGATGGCTTCCTTGTA-3' and reverse, 5'-ACCTCGTGGAGACGCTTTACAT-3'; NOQ1 forward, 5'-ACGACAACGGTCCTTTCCAGA-3' and reverse, 5'-CAGAAACGCAGGATGCCACT-3'; GAPDH forward, 5'-GGACCTGACCTGCCGTCTAG-3' and reverse, 5'-GTA GCCCAGGATGCCCTTGA-3'. The following thermocycling conditions were used for the qPCR: Initial denaturation at $95^{\circ} \mathrm{C}$ for $5 \mathrm{~min} ; 40$ cycles of denaturation at $95^{\circ} \mathrm{C}$ for $10 \mathrm{sec}$, annealing at $58^{\circ} \mathrm{C}$ for $45 \mathrm{sec}$ and elongation at $72^{\circ} \mathrm{C}$ for $1 \mathrm{~min}$; and a final extension at $72^{\circ} \mathrm{C}$ for $5 \mathrm{~min}$. The relative expressions of Nrf2, HO-1 and NOQ1 mRNA were quantified using the $2^{-\Delta \Delta \mathrm{Cq}}$ method and normalized to GAPDH (32). Each sample was measured in triplicate.

Flow cytometric analysis of cell apoptosis. H9c2 cells were seeded at $5 \times 10^{5}$ cells/well into a 6 -well plate and treated as described above for $24 \mathrm{~h}$ prior to digestion with $0.05 \%$ trypsinEDTA to harvest the cells. In total, $1 \times 10^{5}$ treated cells were resuspended in binding buffer (500 $\mu \mathrm{l})$, and incubated with Annexin V-FITC (5 $\mu \mathrm{l})$ and propidium iodide (PI; $5 \mu \mathrm{l})$ for $15 \mathrm{~min}$ at room temperature according to the manufacturer's instructions (BD Pharmingen; BD Biosciences). Subsequently, apoptosis was analyzed by flow cytometry (BD FACSCalibur; BD Biosciences) with CellQuest software (version 3.3; BD Biosciences). Apoptotic cells were counted and represented as a percentage of the total cell count.

ROSgenerationanalysis. The measurementofintracellularROS production was dependent on the ROS-mediated conversion of non-fluorescent DCFH-DA to DCFH. Following treatment, H9c2 cells were harvested and co-incubated with serum-free medium containing DCFH-DA $(50 \mu \mathrm{mol} / \mathrm{l})$ for $20 \mathrm{~min}$ at $37^{\circ} \mathrm{C}$. Subsequently, the cells were rinsed three times with PBS, and the fluorescence intensity in each group was measured by flow cytometry (BD FACSCalibur; BD Biosciences) with CellQuest software (version 3.3;BD Biosciences).

$L D H$ release, malondialdehyde (MDA) content, superoxide dismutase (SOD) and glutathione peroxidase (GSH-Px) activity measurement. LDH release and MDA content were measured using LDH activity assay kit (cat. no. C0017) and lipid peroxidation MDA assay kit (cat. no. S0131M; each, Beyotime Institute of Biotechnology), respectively, according to manufacturer's protocol. SOD and GSH-Px activities were detected by SOD (cat. no. A001-3-2) and GSH-Px (cat. no. A005-1-2) assay kits purchased from Nanjing Jiancheng Bioengineering Institute Co., Ltd. according to the manufacturer's instructions. MDA content was determined using the thiobarbituric acid method, SOD activity was measured using the xanthine oxidase method and GSH-Px activity was determined using the dithio-dinitrotoluidine method.

Western blot analysis. Cells were rinsed twice with cold PBS and nuclear and total proteins were extracted using a Cell Nuclear and Cytoplasmic Protein Extraction kit (cat. no. P0027) and cell lysis buffer (cat. no. P0013) for western blots containing protease inhibitors (phenylmethylsulfonyl fluoride; cat. no. ST505) and a protease inhibitor cocktail (cat. no P1009) all from Beyotime Institute of Biotechnology. Protein concentration was measured using the bicinchoninic acid method (Beyotime Institute of Biotechnology). Equal amounts of protein lysate $(30 \mu \mathrm{g})$ were separated by $12 \%$ SDS-PAGE and then transferred to polyvinylidene fluoride membranes [Roche Diagnostics (Shanghai) Co., Ltd.]. Upon blocking in 5\% non-fat milk for $2 \mathrm{~h}$ at room temperature, the membranes were incubated overnight with primary antibodies against AMPK, p-AMPK, Nrf2, Bax, Bcl-2, NOX2 and GAPDH (all dilutions were 1:1,000) at $4^{\circ} \mathrm{C}$. After washing with TBS-Tween 20 solution, the membranes were then incubated with secondary antibodies (cat. no. 7074) for $2 \mathrm{~h}$ at $37^{\circ} \mathrm{C}$ (horseradish peroxidase-conjugated AffiniPure Goat Anti-Rabbit IgG; 1:5,000; Cell Signaling Technology, Inc.). Protein bands were visualized using an enhanced chemiluminescent agent (Beyotime Institute of Biotechnology). The results were determined by GraphPad Prism 5.0 software (GraphPad Software, Inc.), and the expression of total protein was expressed relative to that of GAPDH. The expression of Nrf2 in the nucleus was expressed relative to that of histone $\mathrm{H} 3$.

Statistical analysis. All data are represented as the mean \pm SD. Differences between groups were determined by one-way ANOVA followed by Tukey's post hoc test. $\mathrm{P}<0.05$ was considered to indicate a statistically significant difference.

\section{Results}

Schizandrin B inhibits myocardial injury following myocardial $H / R$ in $H 9 c 2$ cells. To investigate the cardioprotective effects of schizandrin B against MIRI in vitro, cytotoxicity and apoptosis after $H / R$ injury in the presence or absence of schizandrin B were examined. The CCK-8 assay results showed that schizandrin B pretreatment significantly increased cell viability compared with $H / R$ treatment (Fig. 1A). The LDH release results revealed that $\mathrm{H} / \mathrm{R}$ induced the upregulation of LDH release, which was reversed by schizandrin B pretreatment (Fig. 1B). Additionally, annexin V-FITC/PI double staining results indicated that $\mathrm{H} / \mathrm{R}$ resulted in a significant increase in the apoptosis rate compared with controls, which was reversed by schizandrin B treatment (Fig. 1C). Schizandrin B treatment alone had no effects on cell survival or apoptosis compared with controls. These results indicated that schizandrin B protects $\mathrm{H} 9 \mathrm{c} 2$ cells against $H / R$ injury.

Schizandrin B attenuates oxidative stress in $\mathrm{H} 9 \mathrm{c} 2$ cells subjected to $H / R$. Evidence has shown the contribution of oxidative stress to MIRI (8). Thus, the present study investigated the effects of schizandrin $\mathrm{B}$ on oxidative stress-related biomarkers and the antioxidant defense system in H/R-treated H9c2 cells. As illustrated in Fig. 2, compared with the control groups, H/R treatment increased intracellular ROS generation (Fig. 2A), MDA content (Fig. 2B) and NOX2 expression (Fig. 2C and D) in H9c2 cells. However, these effects were reversed by schizandrin B pretreatment. In addition, compared with the control groups, $\mathrm{H} / \mathrm{R}$ led to a decrease in the enzymatic activities of SOD (Fig. 2E) and GSH-Px (Fig. 2F), which was prevented by schizandrin B pretreatment. Schizandrin B treatment alone had no effect on oxidative stress. Taken together, 
A
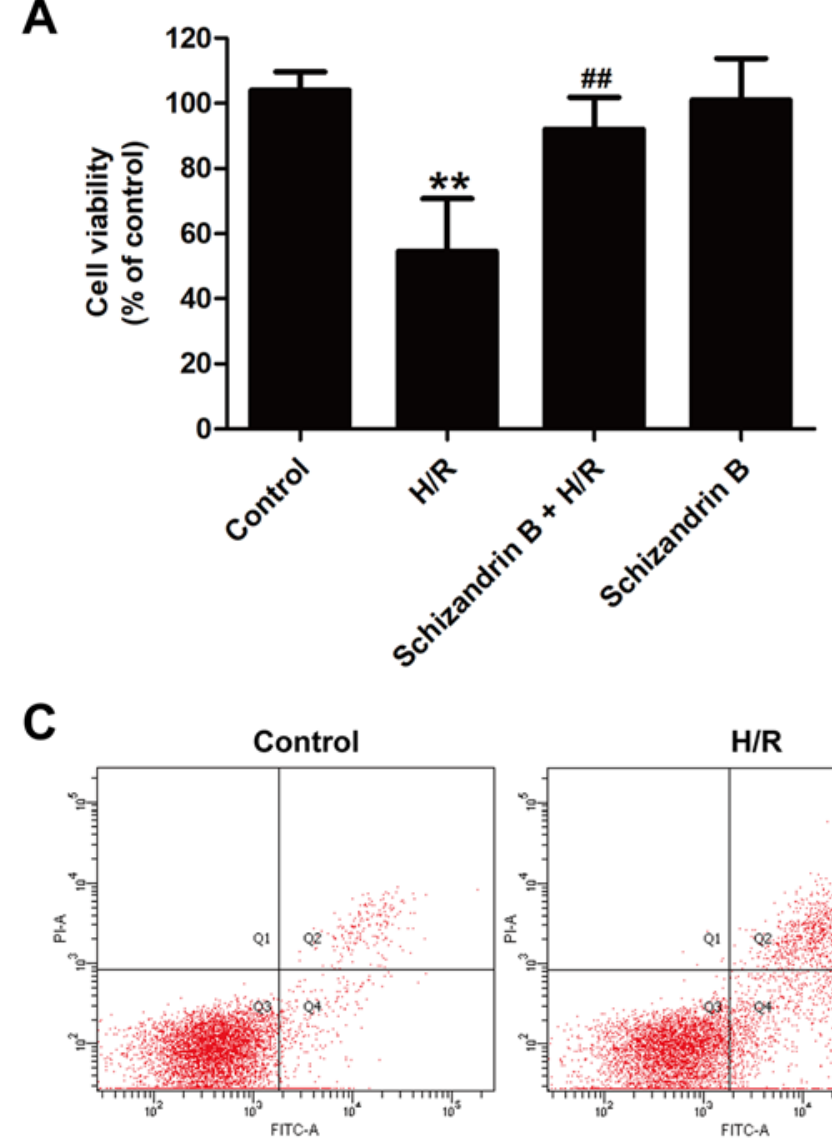

Schizandrin B + H/R

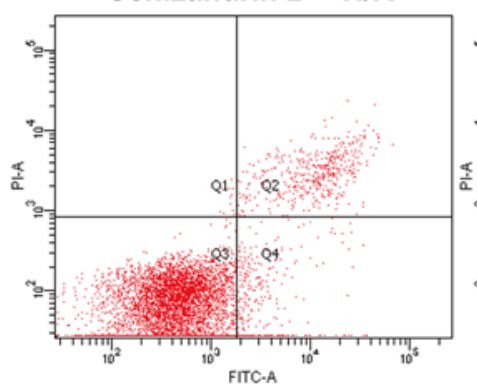

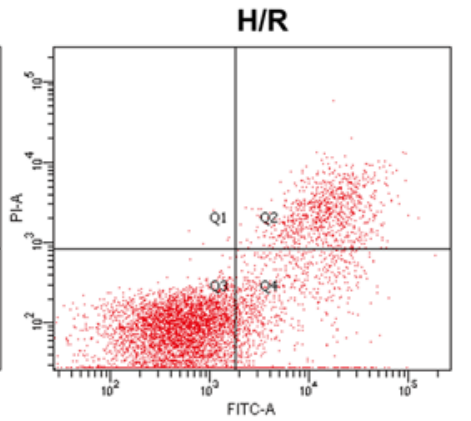

Schizandrin B

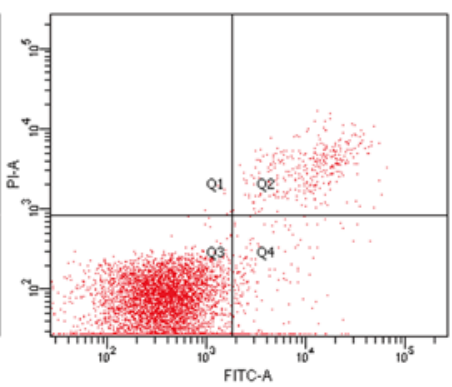

B

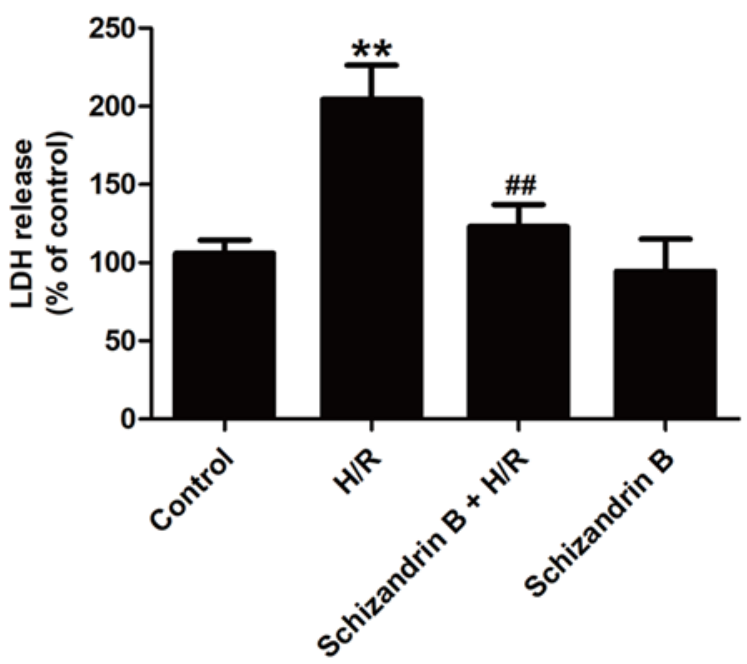

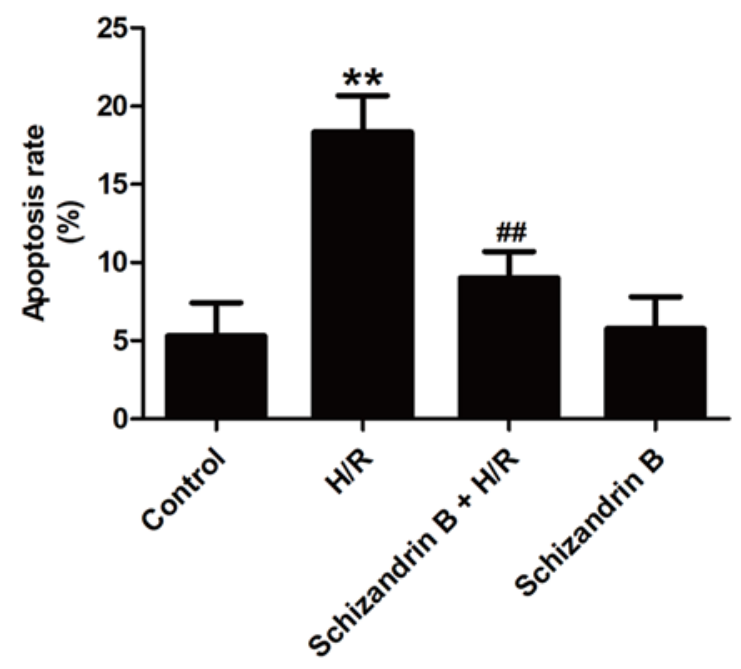

Figure 1. Cardioprotective effects of schizandrin B in H9c2 cells with H/R injury H9c2 cells were treated with schizandrin B $(20 \mu \mathrm{M})$ prior to H $(6 \mathrm{~h}) / \mathrm{R}(12 \mathrm{~h})$. (A) Cell viability was determined using a Cell Counting Kit-8 assay. The results were expressed as the percentage of untreated control. (B) LDH release was measured using an LDH cytotoxicity assay kit. The results were expressed as the fold-change of the untreated control. (C) The apoptosis rate was determined by Annexin V-FITC/PI double staining followed by flow cytometry. Values are expressed as the mean \pm standard deviation from three independent experiments. ${ }^{* *} \mathrm{P}<0.01$ vs. control group; ${ }^{\# \#} \mathrm{P}<0.01$ vs. $\mathrm{H} / \mathrm{R}$ group. H/R, hypoxia/reperfusion; LDH, lactate dehydrogenase; PI, propidium iodide.

these data indicated that the cardioprotection of schizandrin B is antioxidant-dependent.

Schizandrin $B$ reduces the inflammatory response in $H 9 c 2$ cells after H/R injury. Eliminating ROS production is important for controlling inflammatory response (33). To assess the anti-inflammatory effects of schizandrin B in MIRI, the levels of pro-inflammatory and anti-inflammatory cytokines in $\mathrm{H} 9 \mathrm{c} 2$ cells were evaluated. As shown in Fig. 3, compared with the control groups, H/R treatment significantly increased the levels of pro-inflammatory IL-1 $\beta$ (Fig. 3A), TNF- $\alpha$ (Fig. 3B), IL-6 (Fig. 3C) and IL-8 (Fig. 3D), while these effects were all reversed by schizandrin $\mathrm{B}$. In addition, the release of antiinflammatory cytokines TGF- $\beta$ and IL-10 was also detected.
$\mathrm{H} / \mathrm{R}$ significantly reduced the levels of TGF- $\beta$ (Fig. 3E) and IL-10 (Fig. 3F) compared with the control groups. However, these effects were also effectively reversed by schizandrin B. Schizandrin B treatment alone did not affect the inflammatory response. Taken together, these results demonstrated that schizandrin $\mathrm{B}$ inhibits the inflammatory response in H/R-treated H9c2 cells.

Schizandrin $B$ activates the Nrf2 signaling pathway in $H / R$-treated H9c2 cells. Nrf2, a basic leucine zipper transcription factor, modulates the levels of numerous ROS detoxifying and antioxidant genes such as NQO-1 and HO-1 (20,21). To investigate whether schizandrin $\mathrm{B}$ protects $\mathrm{H} 9 \mathrm{c} 2$ cells from $\mathrm{H} / \mathrm{R}$ injury by activating the Nrf2 signaling pathway, Nrf2 
A

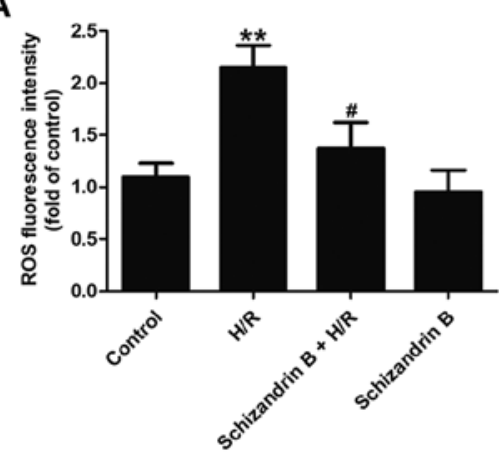

D

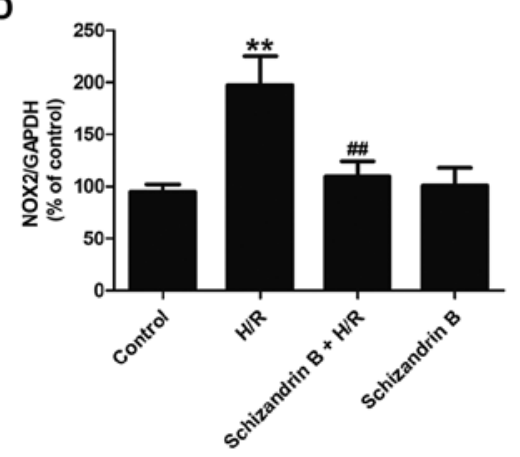

B

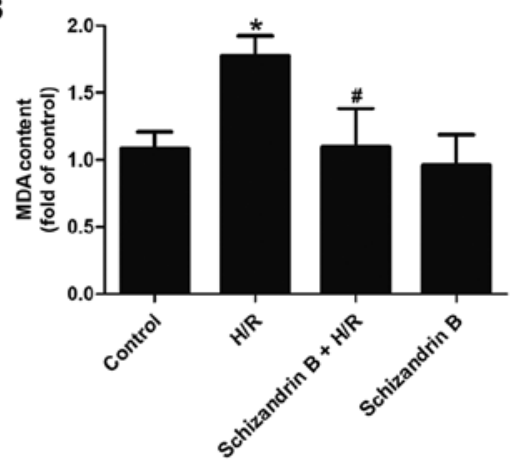

E

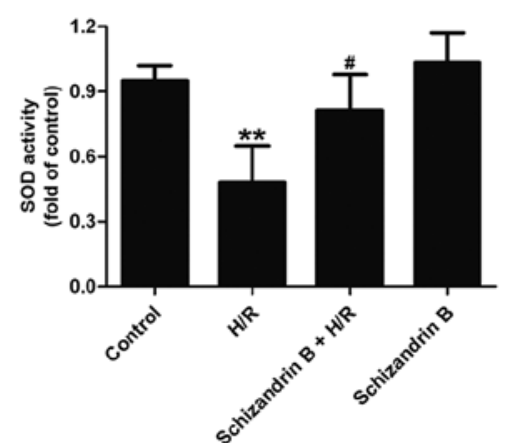

C

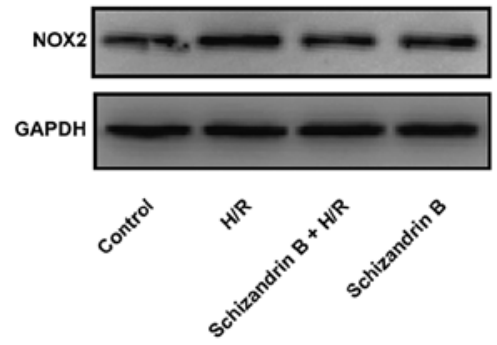

$\mathbf{F}$

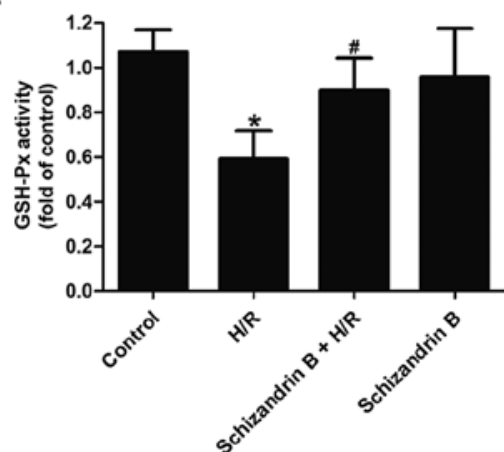

Figure 2. Antioxidative effects of schizandrin B in H/R-treated H9c2 cells. H9c2 cells were treated with schizandrin B $(20 \mu \mathrm{M})$ prior to $\mathrm{H}(6 \mathrm{~h}) / \mathrm{R}(12 \mathrm{~h})$. (A) Intracellular ROS generation was evaluated using a 2',7'-dichlorofluorescein acetyl acetate kit followed by flow cytometry analysis. (B) MDA content was determined using a lipid peroxidation MDA assay kit. (C) The expression of NOX2 was measured by western blotting. (D) Densitometric analysis for NOX2 expression normalized to GAPDH. (E) SOD activity was detected using SOD assay kits. (F) GSH-Px activity was measured using GSH-Px assay kits. Values are expressed as the mean \pm standard deviation from three independent experiments. ${ }^{*} \mathrm{P}<0.05$ and ${ }^{* *} \mathrm{P}<0.01$ vs. control group; ${ }^{\#} \mathrm{P}<0.05$ and ${ }^{\# \#} \mathrm{P}<0.01$ vs. $\mathrm{H} / \mathrm{R}$ group. H/R, hypoxia/reperfusion; ROS, reactive oxygen species; MDA, malondialdehyde; NOX2,NADPH oxidase 2; SOD, superoxide dismutase; GSH-Px, glutathione peroxidase.

A

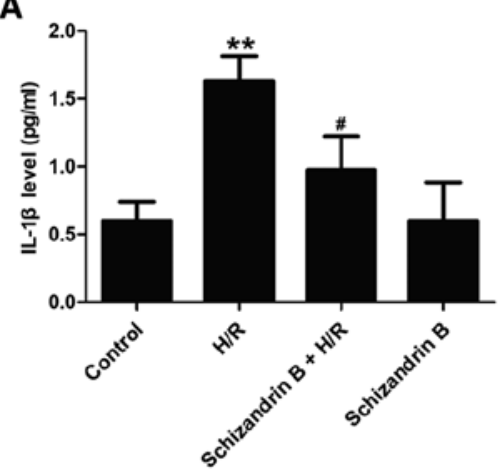

D

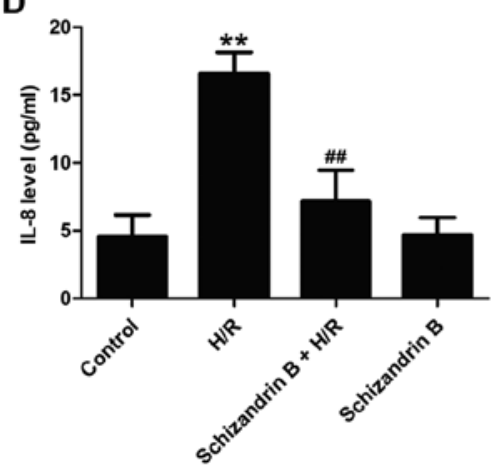

B

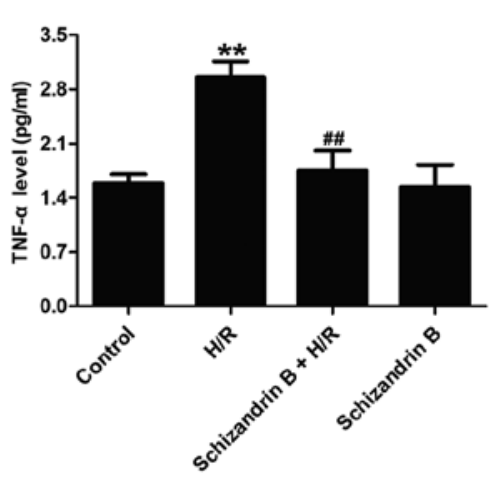

$\mathbf{E}$

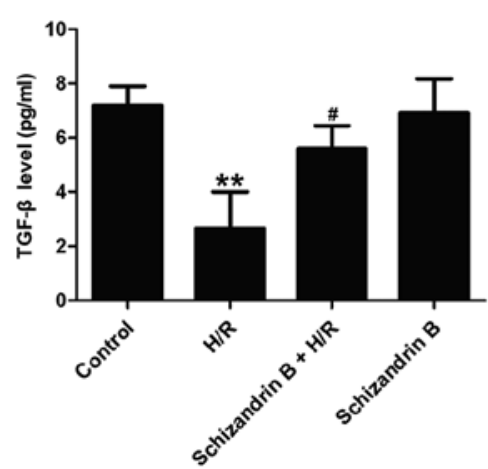

C

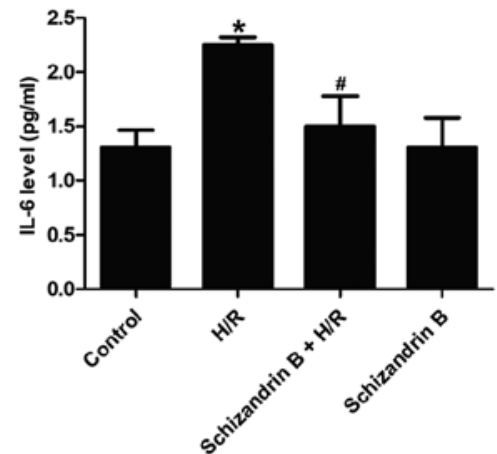

$\mathbf{F}$

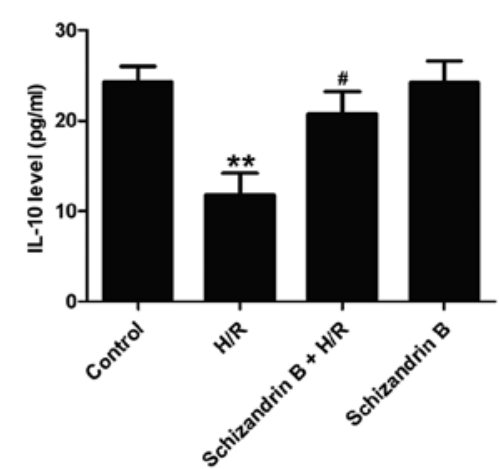

Figure 3. Anti-inflammatory effect of schizandrin B in H/R-treated H9c2 cells. H9c2 cells were treated with schizandrin B $(20 \mu \mathrm{M})$ prior to $\mathrm{H}(6 \mathrm{~h}) / \mathrm{R}(12 \mathrm{~h})$. The levels of (A) IL-1 $\beta$, (B) TNF- $\alpha$, (C) IL-6, (D) IL-8, (E) TGF- $\beta$ and (F) IL-10 were measured by ELISA. Values are expressed as the mean \pm standard deviation from three independent experiments. ${ }^{*} \mathrm{P}<0.05$ and ${ }^{* * *} \mathrm{P}<0.01$ vs. control group; ${ }^{*} \mathrm{P}<0.05$ and ${ }^{\# \#} \mathrm{P}<0.01$ vs. H/R group. H/R, hypoxia/reperfusion; IL, interleukin; TNF- $\alpha$, tumor necrosis factor- $\alpha$; TGF- $\beta$, transforming growth factor- $\beta$. 

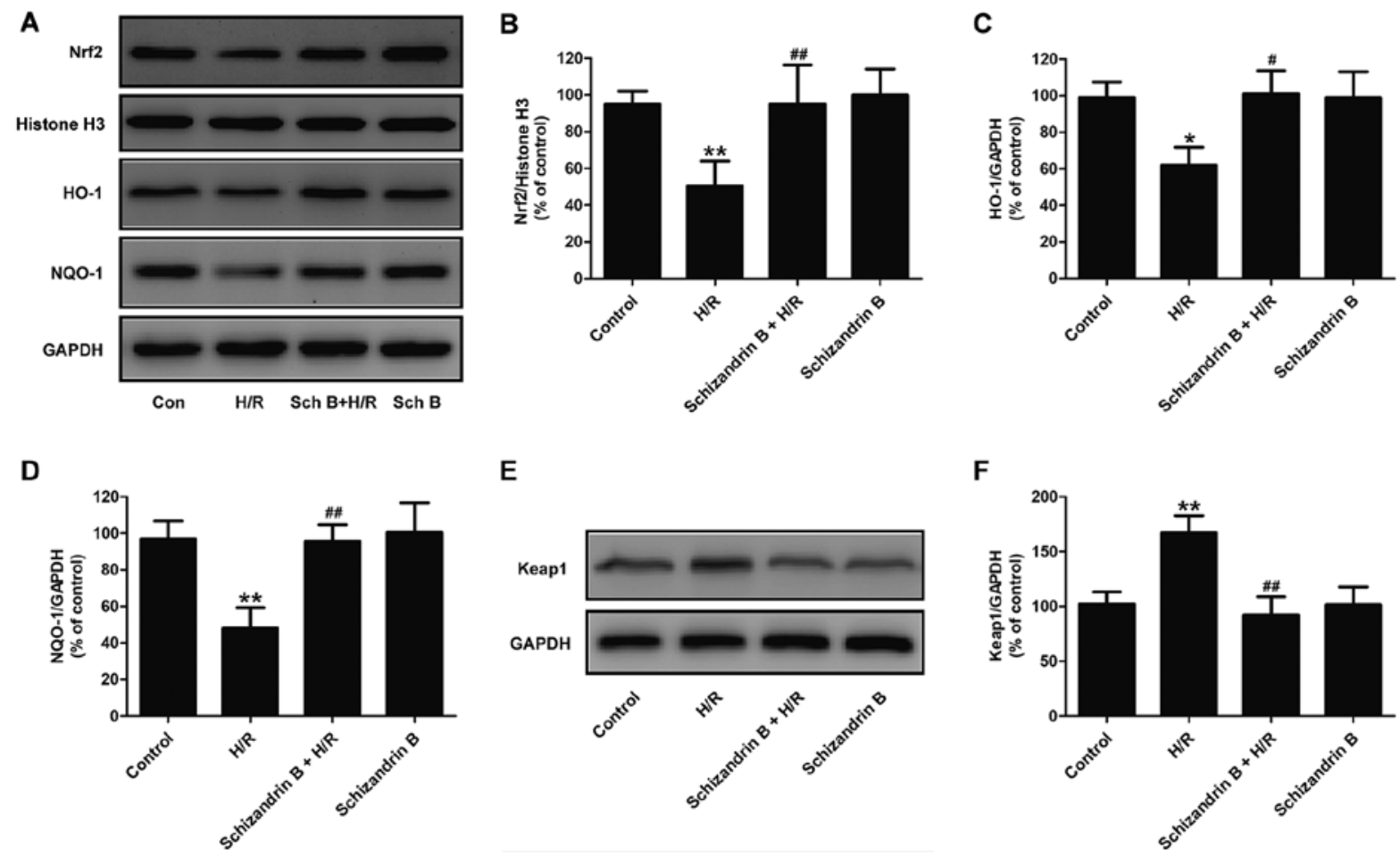

Figure 4. Schizandrin B activates the Nrf2 signaling pathway in H/R-treated H9c2 cells. H9c2 cells were treated with schizandrin B (20 $\mu \mathrm{M})$ prior to $\mathrm{H}(6 \mathrm{~h}) / \mathrm{R}$ (12 h). (A) The expression of Nrf2, NQO-1 and HO-1 was measured by western blotting. (B) Densitometric analysis for Nrf2 expression normalized to histone H3. (C) Densitometry for HO-lexpression normalized to GAPDH. (D) Densitometry for NQO-lexpression normalized to GAPDH. (E) The expression of Keap1 was measured by western blotting. (F) Densitometric analysis for Keap1 expression normalized to GAPDH. Values are expressed as the mean \pm standard deviation from three independent experiments. " $\mathrm{P}<0.05$ and ${ }^{* *} \mathrm{P}<0.01$ vs. control group; ${ }^{*} \mathrm{P}<0.05$ and ${ }^{\# \#} \mathrm{P}<0.01 \mathrm{vs}$. H/R group. H/R, hypoxia/reperfusion; Nrf2, nuclear factor erythroid 2-related factor 2; HO-1, heme oxygenase-1; NQO-1, NAD(P)H: Quinone oxidoreductase; Keap1, Kelch-like ECH-associated protein 1.

expression and transcription efficiency of Nrf2 were assessed. Western blot analysis results (Fig. 4A) indicated that H/R significantly reduced the expression of Nrf2 (Fig. 4B) in the nucleus compared with controls, while schizandrin B pretreatment reversed this effect. The present study further evaluated HO-1 and NQO-1 expression to investigate the activation of $\mathrm{Nrf} 2$ signaling. The results showed that $\mathrm{H} / \mathrm{R}$ significantly decreased the expression of HO-1 (Fig. 4A and C) and NQO-1 (Fig. 4A and D) in H9c2 cells compared with the control groups. However, these effects were reversed by schizandrin $B$ pretreatment. In addition, H/R-induced upregulation of Keap1, an Nrf2 repressor that leads to Nrf2 ubiquitination and degradation, was significantly attenuated by schizandrin B (Fig. 4E and F). Schizandrin B treatment alone had no impact on the Nrf2 signaling pathway. Taken together, these results indicated that schizandrin B might exert beneficial effects in H/R injury by activating the Nrf2 signaling pathway.

Schizandrin B exhibits cardioprotective effects in an Nrf2dependent manner in H/R-treated H9c2 cells. si-Nrf2 was used to knockdown Nrf2 expression and further verify the role of Nrf2 signaling in schizandrin B-induced cardioprotection. Knockdown efficiency was first determined by RT-qPCR, and the results showed that si-Nrf2 transfection significantly reduced the levels of Nrf2 mRNA compared with scramble siRNA transfection (Fig. 5A). The mRNA levels of the Nrf2-dependent antioxidant genes HO-1 and NQO-1 were also significantly reduced by si-Nrf2 transfection compared with scramble siRNA transfection in schizandrin B-treated cells (Fig. 5B). These results indicated that inhibition of the Nrf2 signaling pathway was induced by si-Nrf2 transfection. In addition, si-Nrf2 transfection inhibited schizandrin $\mathrm{B}$-induced upregulation of cell viability in $\mathrm{H} / \mathrm{R}$ compared with scramble siRNA transfection (Fig. 5C). Si-Nrf2 transfection also reversed the schizandrin B-induced decrease in apoptosis rate compared with scrambled siRNA transfection (Fig. 5D and E). Furthermore, si-Nrf2 transfection blocked the anti-inflammatory effect of schizandrin B in H/R injury, as evidenced by the fact that si-Nrf2 transfection reversed schizandrin B-induced downregulation of pro-inflammatory cytokines (IL-1 $\beta$, TNF- $\alpha$ and IL-8) and the upregulation of the anti-inflammatory cytokine IL-10 (Fig. 5F). Furthermore, si-Nrf2 transfection blocked the decrease in ROS generation (Fig. 5G) and the increase in SOD and GSH-Px (Fig. 5H) activities induced by schizandrin B in H/R-treated H9c2 cells compared with scramble siRNA transfection. Taken together, these data indicated that schizandrin B protects $\mathrm{H} 9 \mathrm{c} 2$ cells against $H / R$ injury via enhancing Nrf2 signaling pathway activation.

Nrf2-dependent cardioprotective effects of schizandrin B are mediated by the AMPK pathway. Previous studies demonstrated that AMPK regulates a variety of biological processes and is a highly effective therapeutic target for protecting against MIRI $(21,34)$. Notably, AMPK can stimulate the nuclear accumulation of Nrf2 $(26,27)$. The present study further investigated whether AMPK may be responsible for the activation of Nrf2 in the protective effect of schizandrin B. The results 
A

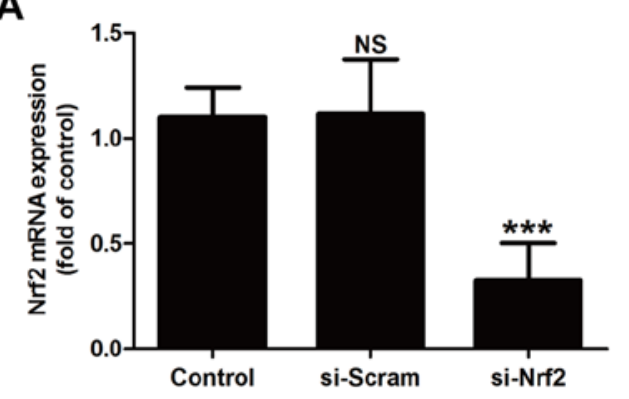

C

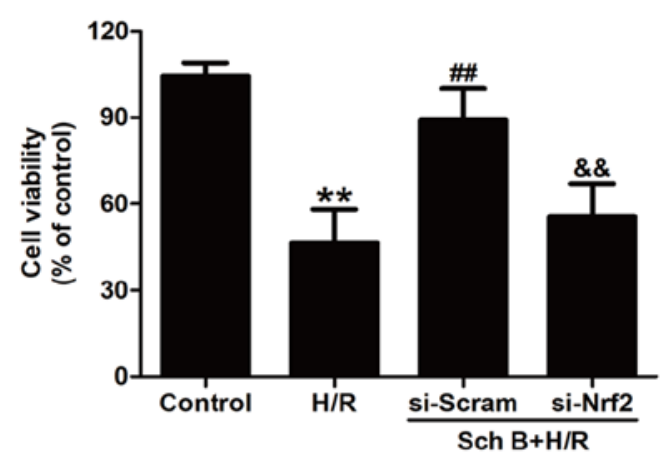

E
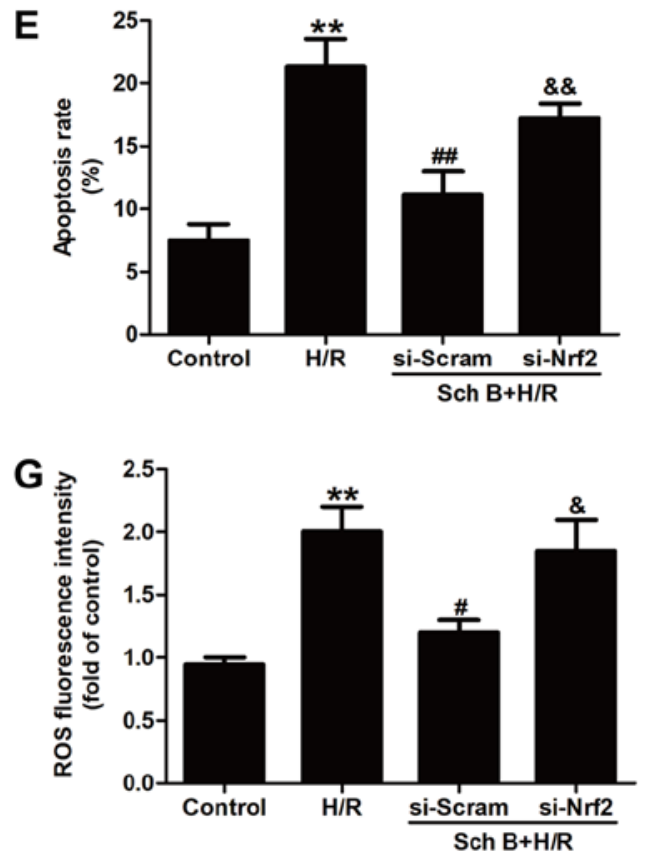

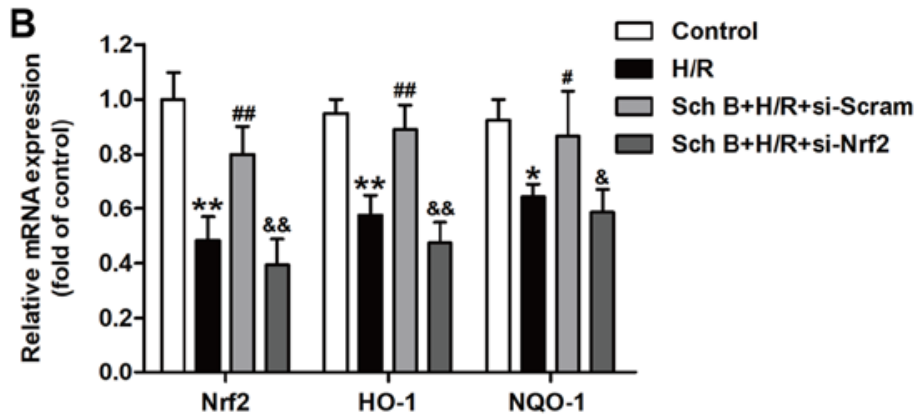

D
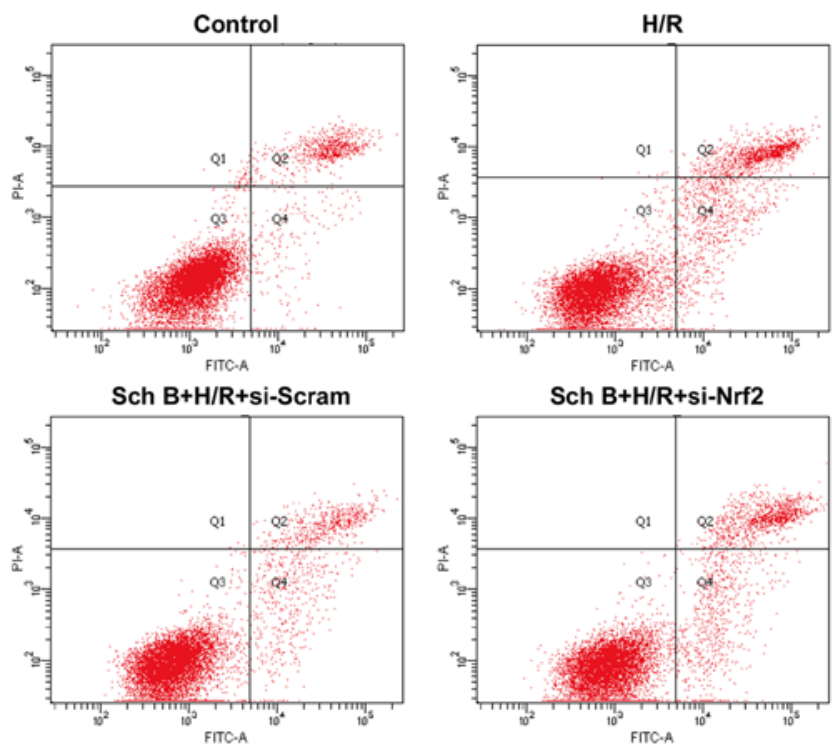

$\mathbf{F}$

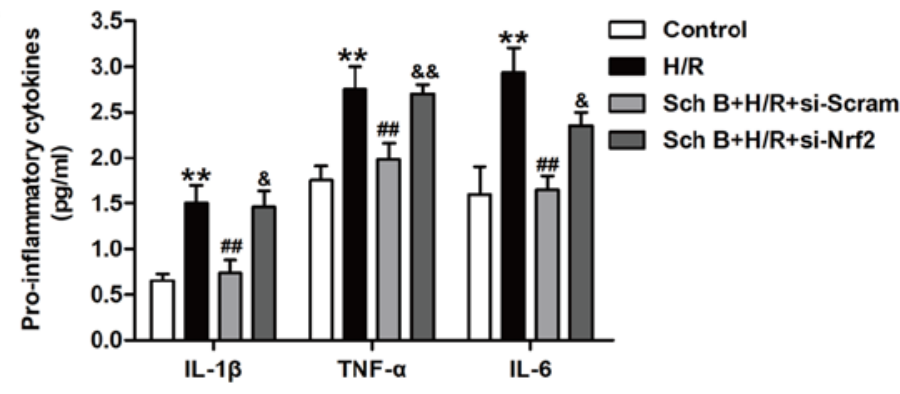

H

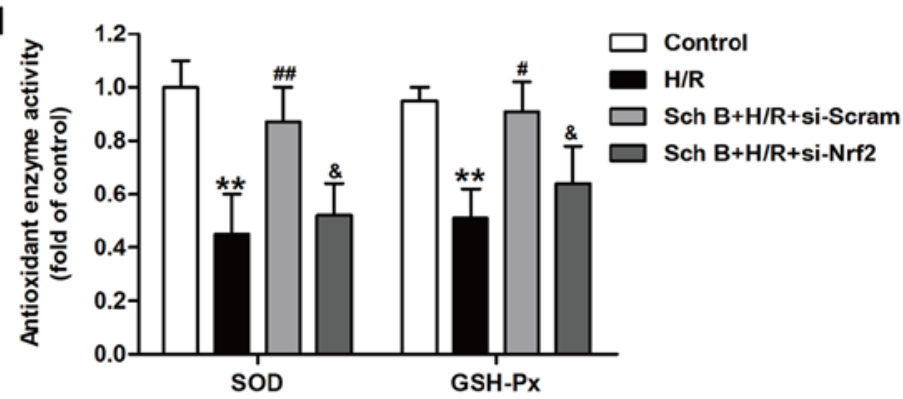

Figure 5. Nrf2 signaling pathway mediates the cardioprotection of schizandrin B in H/R-treated H9c2 cells. H9c2 cells were transfected with si-Nrf2 or si-Scram for $48 \mathrm{~h}$, followed by treatment with schizandrin B $(20 \mu \mathrm{M})$ prior to $\mathrm{H}(6 \mathrm{~h}) / \mathrm{R}(12 \mathrm{~h})$. (A) The levels of Nrf2 mRNA were in si-Nrf2- and si-Scram-transfected cells. NSP $>0.05$ vs. control group; ${ }^{* * *} \mathrm{P}<0.001$ vs. si-Scram group. (B) The levels of Nrf2, HO-1 and NQO-1 mRNA were measured by reverse transcription-quantitative PCR.(C) Cell viability was determined by performing a Cell Counting Kit-8 assay. The results were expressed as a percentage of the untreated control.(D) The apoptosis rate was determined by Annexin V-FITC/PI double staining followed by flow cytometry. (E) Quantitative analysis of apoptosis rates. (F) The levels of proinflammatory cy tokines were detected by ELISA.(G) Intracellular ROS generation was assessed using a 2', 7'-dichlorofluorescein acetyl acetate kit followed by flow cytometry.(H) SOD and GSH-Px activities were detected with SOD and GSH-Px assay kits, respectively. Values are expressed as the mean \pm standard deviation from three independent experiments. ${ }^{*} \mathrm{P}<0.05$ and ${ }^{* *} \mathrm{P}<0.01$ vs.control group; ${ }^{*} \mathrm{P}<0.05$ and ${ }^{\# /} \mathrm{P}<0.01 \mathrm{vs.H} / \mathrm{R}$ group ${ }^{\&} \mathrm{P}<0.05$ and ${ }^{\text {\&\&}} \mathrm{P}<0.01$ vs. schizandrin $\mathrm{B}+\mathrm{H} / \mathrm{R}+$ si-Nrf2 group. H/R, hypoxia/reperfusion; Nrf2, nuclear factor erythroid 2-related factor 2; HO-1, heme oxygenase-1; NQO-1, NAD(P)H: Quinone oxidoreductase; si-Nrf2, Nrf2-specific small interfering RNA; si-Scram, scrambled siRNA; Sch B, schizandrin B; IL, interleukin; TNF- $\alpha$, tumor necrosis factor- $\alpha$; SOD, superoxide dismutase; GSH-Px, glutathione peroxidase; PI, propidium iodide. 
A

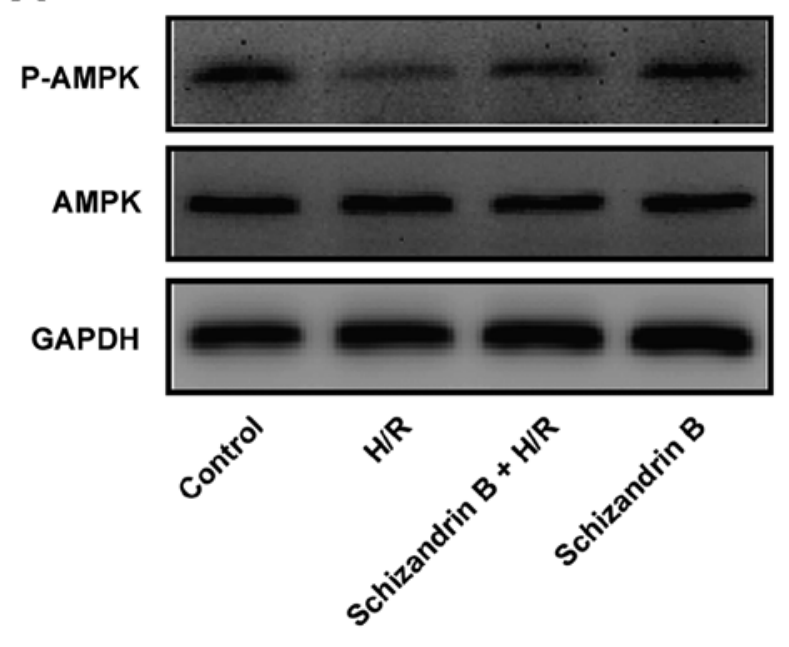

B

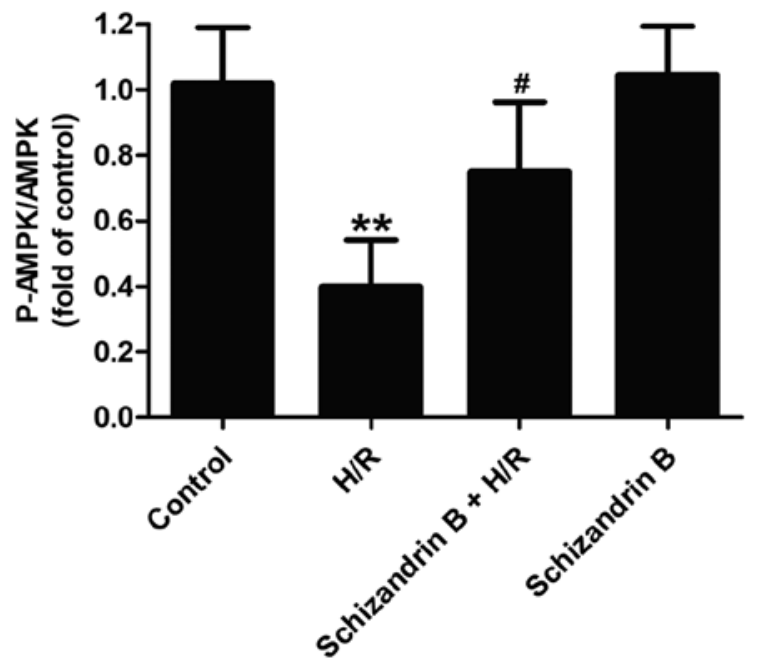

C

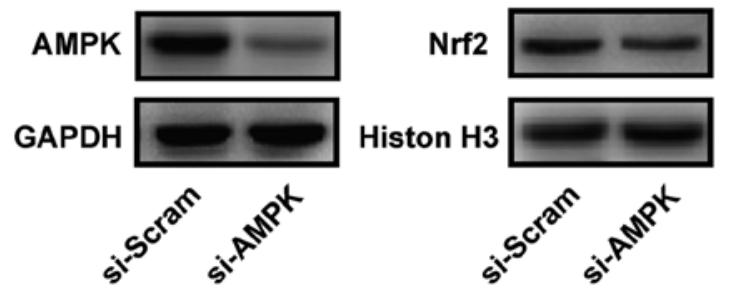

$\mathbf{E}$

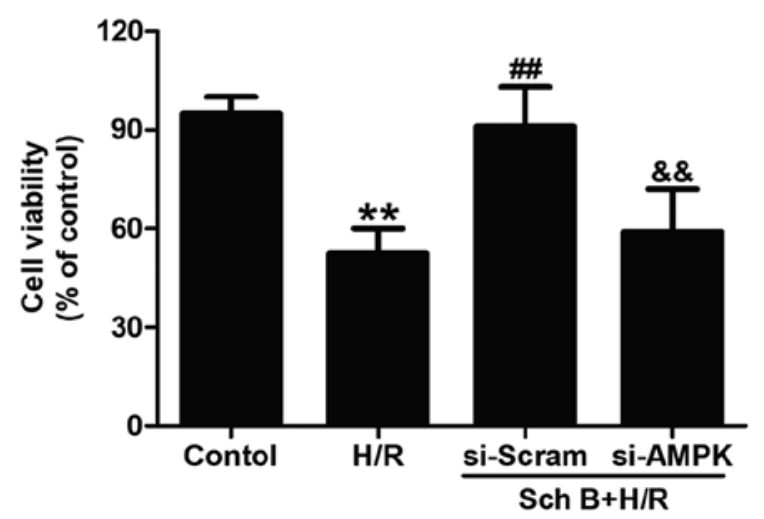

D

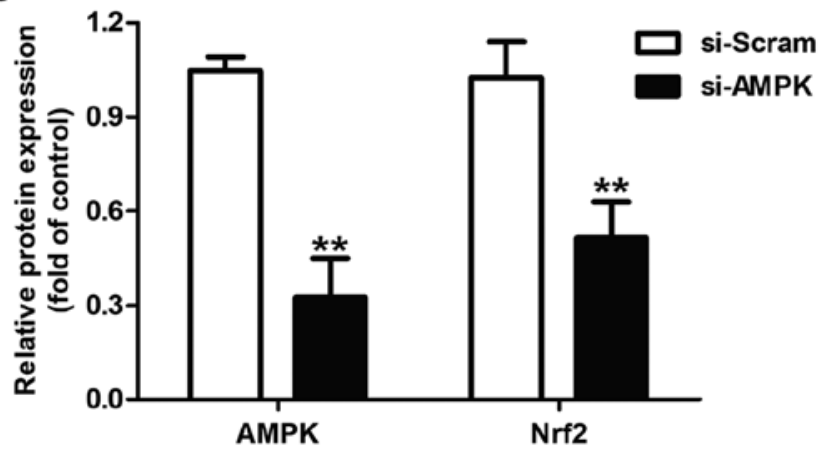

$\mathbf{F}$

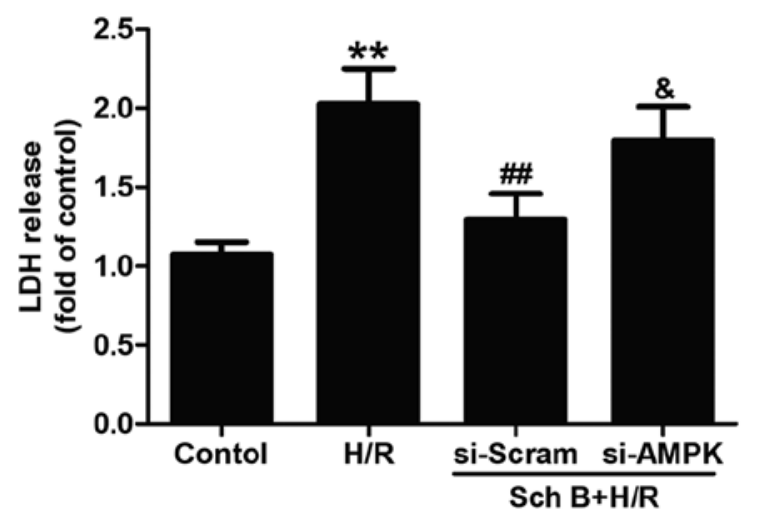

Figure 6. AMPK pathway contributes to Nrf2-dependent cardioprotective effects of schizandrin B in H/R-treated H9c2 cells. H9c2 cells were treated with schizandrin B $(20 \mu \mathrm{M})$ prior to $\mathrm{H}(6 \mathrm{~h}) / \mathrm{R}(12 \mathrm{~h})$. (A) The expression of P-AMPK and AMPK were measured by western blotting. (B) Densitometric analysis for P-AMPK expression normalized to total AMPK. Values are expressed as the mean \pm standard deviation from three independent experiments. ${ }^{* *} \mathrm{P}<0.01$ vs. control group; ${ }^{\text {P }}<0.05 v s$. H/R group. H9c2 cells weretransfected with si-AMPK or si-Scram for $48 \mathrm{~h}$. (C) The expression of AMPK and Nrf2 was analyzed by western blot analysis. (D) Densitometric analysis of AMPK and Nrf2 expression. ${ }^{* *} \mathrm{P}<0.01$ vs.si-Scram groups. H9c2 cells were transfected with si-AMPK or si-Scram for $48 \mathrm{~h}$, followed by treatment with schizandrin B $(20 \mu \mathrm{M})$ prior to $\mathrm{H}(6 \mathrm{~h}) / \mathrm{R}(12 \mathrm{~h})$. (E) Cell viability in the different groups was evaluated by a Cell Counting Kit-8 assay. (F) LDH release was measured using an LDH cytotoxicity assay kit. Values are expressed as the mean \pm standard deviation from three independent experiments. ${ }^{* *} \mathrm{P}<0.01$ vs. control group; ${ }^{\# \#} \mathrm{P}<0.01 \mathrm{vs} . \mathrm{H} / \mathrm{R}$ group; ${ }^{\&} \mathrm{P}<0.05$ and ${ }^{\&} \mathrm{P}<0.01$ vs. schizandrin $\mathrm{B}+\mathrm{H} / \mathrm{R}+\mathrm{si}-\mathrm{AMPK}$ group. AMPK, adenosine monophosphate-activated protein kinase; p-AMPK, phospho-AMPK; H/R, hypoxia/reperfusion; Nrf2, nuclear factor erythroid 2-related factor 2; si-AMPK, AMPK-specific small interfering RNA; si-Nrf2, Nrf2-specific siRNA; si-Scram, scrambled siRNA; Sch B, schizandrin B; LDH, lactate dehydrogenase.

showed that schizandrin B pretreatment increased the phosphorylation of AMPK in H/R-treated H9c2 cells compared with the H/R group (Fig. 6A and B). Cells were transfected with si-AMPK, and the western blot results revealed that si-AMPK transfection significantly reduced the expression of AMPK compared with scrambled siRNA transfection 
(Fig. 6C and D). si-AMPK transfection also significantly decreased the expression of $\mathrm{Nrf} 2$ (Fig. 6C and D) compared with scrambled siRNA transfection, indicating that inhibition of the AMPK/Nfr2 signaling pathway was induced by AMPK knockdown. Schizandrin B-mediated increased cell viability (Fig. 6E) and decreased LDH release (Fig. 6F) were reversed by si-AMPK transfection. These results demonstrated that the cardioprotection of schizandrin B in MIRI is dependent on the AMPK/Nfr2 signaling pathway.

\section{Discussion}

The cytoprotective function of schizandrin B in MIRI, in which a decrease in oxidation and ER stress-induced apoptosis has been reported $(14,15)$. The beneficial effects of schizandrin B on ameliorating MIRI are dependent on its anti-apoptotic (14) and antioxidative activities (35), and mitochondrial functional enhancement (36). Despite significant advances in the understanding of the mechanisms accounting for the myocardial protective mechanism of schizandrin $B(2,37)$, the protective mechanism of schizandrin B on MIRI remains to be elucidated. The present study aimed to investigate whether schizandrin B protected $\mathrm{H} 9 \mathrm{c} 2$ cells against $\mathrm{H} / \mathrm{R}$ injury via regulating the AMPK/Nrf2 signaling pathway.

Schizandrin B is an active dibenzocyclooctadiene lignan isolated from the fruit of Schisandra chinensis (a traditional Chinese herb), and was found to possess antioxidant, antiapoptotic, anti-inflammatory and cardioprotective activities in vitro and in vivo $(38,39)$. Studies indicated that schizandrin B could improve cardiac function and mitigate myocardial infarct size in a MIRI model via inhibiting cell apoptosis and attenuating oxidative stress $(15,36)$. Consistent with these studies, our findings also revealed that schizandrin B pretreatment mitigated H/R-induced cytotoxicity and apoptosis.

There are several mechanisms involved in the development of MIRI, including increased oxidative stress and inflammatory response $(40,41)$. Oxidative stress, which is an imbalance between endogenous ROS generation and antioxidant systems, is involved in the etiology of $\mathrm{I} / \mathrm{R}$-induced myocardial injury $(8,42)$ and inflammatory response $(33)$. The participation of oxidative stress and inflammation in the pathologies of MIRI has been reported $(43,44)$. Recent evidence has suggested that certain drugs, such as lipopolysaccharide and myeloperoxidase, that serve anti-oxidative and antiinflammatory functions are considered a therapeutic strategy for limiting MIRI $(39,44)$. The present results showed that schizandrin B pretreatment significantly reduced oxidative stress in H/R-treated H9c2 cells, as evidenced by the decrease in intracellular ROS generation, lipid peroxide (MDA) levels and NOX2 expression, and the increase in antioxidant enzymatic activities, including SOD and GSH-Px, in H9c2 cells. Inflammation is a hallmark of MIRI (45). Previous studies have confirmed that the imbalance between pro-inflammatory (IL-1 $\beta$, TNF- $\alpha$, IL-6 and IL-18) and anti-inflammatory cytokines (TGF- $\beta$, IL-10 and IL-13) contribute to the development of MIRI $(46,47)$. The results of the present study found that schizandrin $B$ attenuated $\mathrm{H} / \mathrm{R}$-induced upregulation of pro-inflammatory cytokines, including IL-1 $\beta$, TNF- $\alpha$, IL-6 and IL-8, and the downregulation of anti-inflammatory cytokines, including TGF- $\beta$ and IL-10, in H9c2 cells, indicating the inhibition of schizandrin B on inflammatory response. These above results are consistent with the anti-oxidant and anti-inflammatory activities of schizandrin B reported in previous studies $(1,29)$. Taken together, these results indicated that schizandrin B protects $H 9 c 2$ cells against $H / R$ injury via inhibiting oxidative stress and inflammation.

Nrf2 is known to play a central role in cellular defense against oxidative stress via regulating the transcriptional expression of downstream antioxidant enzymes such as HO-1 and NOQ1 $(22,48)$. Researches confirm that inhibition of the Nrf2 axis exacerbates oxidative stress, inflammation and induced cell apoptosis $(11,49,50)$. Numerous studies have shown that Nrf2 is one of the essential signaling pathways that can mitigate myocardial infarct size and preserve cardiac function following MIRI, which is dependent on the coordinated upregulation of antioxidant, anti-inflammatory and autophagic mechanisms $(25,51)$. Oxidative stressors and several anti-inflammatory traditional Chinese medicines, such as Arctigenin, Nardochinoid $\mathrm{C}$ and azafrin, promote the nuclear translocation of $\mathrm{Nrf} 2$ and activate the transcription of antioxidant genes, including HO-1 and NOQ1, leading to beneficial protection on various diseases (40,52-54). Notably, schizandrin B was also shown to reduce oxidative stress and possess strong anti-inflammatory properties, at least in part via the induction of Nrf2 and Nrf2-driven antioxidant responses (55). In the present study, schizandrin B pretreatment also enhanced Nrf2 expression in the nucleus, and HO-1 and NOQ1 expression in H9c2 cells. Notably, Nfr2 knockdown induced by si-Nrf2 remarkably attenuated schizandrin B-induced inhibition against cytotoxicity, apoptosis, oxidative stress and inflammation in H/R-treated H9c2 cells. These results showed that the Nfr2 signaling pathway contributed to the cardioprotection of schizandrin B in MIRI.

AMPK activation was verified to confer cardioprotection against MIRI by regulating processes such as survival and cellular longevity, apoptosis, inflammation, ROS reduction and mitochondrial function $(17,56,57)$. AMPK activation is known to promote myocardial resistance to I/R and oxidative stress of different magnitudes by upregulating Nrf2 $(58,59)$. However, the role of the AMPK pathway in the beneficial function of schizandrin B remains to be elucidated. In the present study, the results showed that schizandrin B enhanced AMPK activation in H/R-treated $\mathrm{H} 9 \mathrm{c} 2$ cells. In addition, AMPK knockdown induced by si-AMPK transfection reduced the expression of Nfr2, HO-1 and NOQ1, which is consistent with previous studies where inhibition of AMPK by relative inhibitor or specific siRNA decreased Nrf2 expression in MIRI $(59,60)$. Meanwhile, si-AMPK transfection reversed schizandrin B-induced protection on H/R injury. Taken together, these results showed that the Nrf2-dependent cardioprotective effects of schizandrin B are mediated by the AMPK pathway.

In conclusion, the present results demonstrated that schizandrin B protects $H 9 \mathrm{c} 2$ cells against $\mathrm{H} / \mathrm{R}$ injury by suppressing oxidative stress and inflammation. The present results supported the notion that the AMPK/Nrf2 signaling pathway may play a role in the cardioprotection of schizandrin $B$ against MIRI. These results provide a better understanding of the molecular mechanisms associated with the cardioprotection of schizandrin B and may provide a new insight into a better design of myocardial protective agents against MIRI. 
However, since the present experiments were only performed in vitro, in vivo animal experiments and clinical trials with human subjects should be performed in further studies. In addition, the endogenous hydrogen sulfide-regulated Nrf2 signaling pathway was involved in myocardial protection against I/R injury $(61,62)$. It was also confirmed that Nrf2 regulates hundreds of genes, of which many are either directly or indirectly involved in modulating ferroptosis, which serves as a cardioprotective strategy for cardiomyopathy prevention $(63,64)$. Hence, the role of these above related signaling pathways in the cardioprotection of schizandrin B warrants further study. Additionally, since the method of the present study of checking IRI is a two-step process but presented as a final step, it is also worth checking ischemia and reperfusion as two distinct paths in subsequent experiments.

\section{Acknowledgements}

Not applicable.

\section{Funding}

No funding was received.

\section{Availability of data and materials}

The datasets used and/or analyzed during the current study are available from the corresponding author on reasonable request.

\section{Authors' contributions}

BZ and GPL designed and performed the experiments and wrote the manuscript. JJP, LHR and LCL analyzed the data. HMY and ZYW designed the experiments and analyzed the data. SZ performed the experiments. All authors read and approved the final manuscript.

\section{Ethics approval and consent to participate}

Not applicable.

\section{Patient consent for publication}

Not applicable.

\section{Competing interests}

The authors declare that they have no competing interests.

\section{References}

1. Chi HJ, Chen ML, Yang XC, Lin XM, Sun H, Zhao WS, Qi D, Dong JL and Cai J: Progress in therapies for myocardial ischemia reperfusion injury. Curr Drug Targets 18: 1712-1721, 2017.

2. Neri M, Riezzo I, Pascale N, Pomara C and Turillazzi E: Ischemia/reperfusion injury following acute myocardial infarction: A critical issue for clinicians and forensic pathologists. Mediators Inflamm 2017: 7018393, 2017.

3. Zhu N, Cai C, Zhou A, Zhao X, Xiang Y and Zeng C: Schisandrin B prevents hind limb from ischemia-reperfusioninduced oxidative stress and inflammation via MAPK/NF- $\kappa \mathrm{B}$ pathways in rats. Biomed Res Int 2017: 4237973, 2017.
4. Zheng Q, Huang YY, Zhu PC, Tong Q, Bao XY, Zhang QH, Zheng GQ and Wang Y: Ligustrazine exerts cardioprotection in animal models of myocardial ischemia/reperfusion injury: Preclinical evidence and possible mechanisms. Front Pharmacol 9: 729, 2018.

5. Liu J, Wang $\mathrm{H}$ and Li J: Inflammation and inflammatory cells in myocardial infarction and reperfusion injury: A double-edged sword. Clin Med Insights Cardiol 10: 79-84, 2016.

6. Yang CF: Clinical manifestations and basic mechanisms of myocardial ischemia/reperfusion injury. Ci Ji Yi Xue Za Zhi 30: 209-215, 2018.

7. Gielis JF, Beckers PAJ, Briede JJ, Cos P and Van Schil PE: Oxidative and nitrosative stress during pulmonary ischemiareperfusion injury: From the lab to the OR. Ann Transl Med 5: $131,2017$.

8. González -Montero J, Brito R, Gajardo AI and Rodrigo R: Myocardial reperfusion injury and oxidative stress: Therapeutic opportunities. World J Cardiol 10: 74-86, 2018.

9. He F and Zuo L: Redox roles of reactive oxygen species in cardiovascular diseases. Int J Mol Sci 16: 27770-27780, 2015

10. Li ZM, Xu SW and Liu PQ: Salvia miltiorrhizaBurge (Danshen): A golden herbal medicine in cardiovascular therapeutics. Acta Pharmacol Sin 39: 802-824, 2018.

11. Li J,Zheng X, Ma X, Xu X, Du Y, Lv Q, Li X, Wu Y, Sun H, Yu L and Zhang Z: Melatonin protects against chromium(VI)-induced cardiac injury via activating the AMPK/Nrf2 pathway. J Inorg Biochem 197: 110698, 2019.

12. Yu LM, Dong X, Xue XD, Zhang J, Li Z, Wu HJ, Yang ZL, Yang Y and Wang HS: Naringenin improves mitochondrial function and reduces cardiac damage following ischemia-reperfusion injury: The role of the AMPK-SIRT3 signaling pathway. Food Funct 10: 2752-2765, 2019.

13. Xin DQ, Hu ZM, Huo HJ, Yang XJ, Han D, Xing WH, Zhao Y and Qiu QH: Schisandrin B attenuates the inflammatory response, oxidative stress and apoptosis induced by traumatic spinal cord injury via inhibition of p53 signaling in adult rats. Mol Med Rep 16: 533-538, 2017.

14. Zhang W, Sun Z and Meng F: Schisandrin B ameliorates myocardial ischemia/reperfusion injury through attenuation of endoplasmic reticulum stress-induced apoptosis. Inflammation 40: 1903-1911, 2017.

15. Zhao X, Xiang Y,Cai C,Zhou A,Zhu N and Zeng C: Schisandrin B protects against myocardial ischemia/reperfusion injury via the PI3K/Akt pathway in rats. Mol Med Rep 17: 556-561, 2018.

16. Yim TK and Ko KM: Schisandrin B protects against myocardial ischemia-reperfusion injury by enhancing myocardial glutathione antioxidant status. Mol Cell Biochem 196: 151-156, 1999.

17. Steinberg GR and Schertzer JD: AMPK promotes macrophage fatty acid oxidative metabolism to mitigate inflammation: Implications for diabetes and cardiovascular disease. Immunol Cell Biol 92: 340-345, 2014.

18. Zhang M, Yang D, Gong X, Ge P, Dai J, Lin L and Zhang L: Protective benefits of AMP-activated protein kinase in hepatic ischemia-reperfusion injury. Am J Transl Res 9: 823-829, 2017.

19. Daskalopoulos EP, Dufeys C, Beauloye C, Bertrand L and Horman S: AMPK in cardiovascular diseases. Exp Suppl 107: 179-201, 2016.

20. Kanugula AK and Thodeti CK: AMP-activated kinase 'Keaps' ischemia/reperfusion-induced necroptosis under control. Int J Cardiol 259: 168-169, 2018.

21. Qi D and Young LH: AMPK: Energy sensor and survival mechanism in the ischemic heart. Trends Endocrinol Metab 26: 422-429, 2015.

22. da Costa RM, Rodrigues D, Pereira CA, Silva JF, Alves JV, Lobato NS and Tostes RC: Nrf2 as a potential mediator of cardiovascular risk in metabolic diseases. Front Pharmacol 10: $382,2019$.

23. Liu L, Locascio LM and Doré S: Critical role of Nrf2 in experimental ischemic stroke. Front Pharmacol 10: 153, 2019.

24. Schmidlin CJ, Dodson MB, Madhavan L and Zhang DD: Redox regulation by NRF2 in aging and disease. Free Radic Biol Med 134: 702-707, 2019.

25. Shen Y, Liu X, Shi J and Wu X: Involvement of Nrf2 in myocardial ischemia and reperfusion injury. Int J Biol Macromol 125: 496-502, 2019.

26. Zhou F, Wang M, Ju J, Wang Y, Liu Z, Zhao X, Yan Y, Yan S, Luo X and Fang Y: Schizandrin A protects against cerebral ischemia-reperfusion injury by suppressing inflammation and oxidative stress and regulating the AMPK/Nrf2 pathway regulation. Am J Transl Res 11: 199-209, 2019. 
27. Fan $X, L v H$, Wang L, Deng $X$ and $C i X$ : Isoorientin ameliorates APAP-induced hepatotoxicity via activation $\mathrm{Nrf} 2$ antioxidative pathway: The involvement of AMPK/Akt/GSK3 3 . Front Pharmacol 9: 1334, 2018

28. Yang Q, Han L, Li J, Xu H, Liu X, Wang X, Pan C, Lei C, Chen H and Lan X: Activation of Nrf2 by phloretin attenuates palmitic acid-induced endothelial cell oxidative stress via AMPKdependent signaling. J Agric Food Chem 67: 120-131, 2019.

29. Mou Z, Feng Z, Xu Z, Zhuang F, Zheng X, Li X, Qian J and Liang G: Schisandrin B alleviates diabetic nephropathy through suppressing excessive inflammation and oxidative stress. Biochem Biophys Res Commun 508: 243-249, 2019.

30. Wu Y,Li ZC, Yao LQ, Li M and Tang M: Schisandrin B alleviates acute oxidative stress via modulation of the Nrf2/Keap1-mediated antioxidant pathway. Appl Physiol Nutr Metab 44: 1-6, 2019.

31. Cheng J, Wu Q, Lv R, Huang L, Xu B, Wang X, Chen A and He F: MicroRNA-449a inhibition protects H9C2 cells against hypoxia/reoxygenation-induced injury by targeting the Notch-1 signaling pathway. Cell Physiol Biochem 46: 2587-2600, 2018.

32. Livak KJ and Schmittgen TD: Analysis of relative gene expression data using real-time quantitative PCR and the 2(-Delta Delta C(T)) method. Methods 25: 402-408, 2001.

33. Doridot L, Jeljeli M, Chêne C and Batteux F: Implication of oxidative stress in the pathogenesis of systemic sclerosis via inflammation, autoimmunity and fibrosis. Redox Biol 25: 101122, 2019.

34. Park JS, Leem YH,Park JE, Kim DY and Kim HS: Neuroprotective effect of $\beta$-lapachone in MPTP-induced parkinson's disease mouse model: Involvement of astroglial p-AMPK/Nrf2/HO-1 signaling pathways. Biomol Ther (Seoul) 27: 178-184, 2019

35. Chen N and Ko M: Schisandrin B-induced glutathione antioxidant response and cardioprotection are mediated by reactive oxidant species production in rat hearts. Biol Pharm Bull 33: 825-829, 2010

36. Ko KM and Chiu PY: Structural determinants of schisandrin B which enhance mitochondrial functional ability and glutathione status as well as heat shock protein expression in rat hearts and H9c2 cells. Mol Cell Biochem 276: 227-234, 2005.

37. Lakota J: Molecular mechanism of ischemia-reperfusion injury after myocardial infarction and its possible targeted treatment Int J Cardiol 220: 571-572, 2016

38. Leong PK and Ko KM: Schisandrin B: A double-edged sword in nonalcoholic fatty liver disease. Oxid Med Cell Longev 2016: 6171658, 2016

39. Shao M, Yang W and Han G: Protective effects on myocardial infarction model: Delivery of schisandrin B using matrix metalloproteinase-sensitive peptide-modified, PEGylated lipid nanoparticles. Int J Nanomedicine 12: 7121-7130, 2017.

40. Qiu Z, He Y, Ming H, Lei S, Leng $Y$ and Xia ZY: Lipopolysaccharide (LPS) aggravates high glucose- and hypoxia/reoxygenation-induced injury through activating ROS-dependent NLRP3 inflammasome-mediated pyroptosis in H9C2 cardiomyocytes. J Diabetes Res 2019: 8151836, 2019.

41. Yao BJ, He XQ, Lin YH and Dai WJ: Cardioprotective effects of anisodamine against myocardial ischemia/reperfusion injury through the inhibition of oxidative stress, inflammation and apoptosis. Mol Med Rep 17: 1253-1260, 2018.

42. Yang J, Yin HS, Cao YJ, Jiang ZA, Li YJ, Song MC, Wang YF, Wang ZH, Yang R, Jiang YF, et al: Arctigenin attenuates ischemia/reperfusion induced ventricular arrhythmias by decreasing oxidative stress in rats. Cell Physiol Biochem 49: 728-742, 2018.

43. Yang MY, Wang YB, Han B, Yang B, Qiang YW, Zhang Y, Wang Z, Huang X, Liu J, Chen YD, et al: Activation of aldehyde dehydrogenase 2 slows down the progression of atherosclerosis via attenuation of ER stress and apoptosis in smooth muscle cells. Acta Pharmacol Sin 39: 48-58, 2018.

44. Ndrepepa G: Myeloperoxidase-A bridge linking inflammation and oxidative stress with cardiovascular disease. Clin Chim Acta 493: 36-51, 2019.

45. Rohrbach S, Troidl C, Hamm C and Schulz R: Ischemia and reperfusion related myocardial inflammation: A network of cells and mediators targeting the cardiomyocyte. IUBMB Life 67: 110-119, 2015.

46. Vinten-Johansen J, Jiang R, Reeves JG, Mykytenko J, Deneve J and Jobe LJ: Inflammation, proinflammatory mediators and myocardial ischemia-reperfusion Injury. Hematol Oncol Clin North Am 21: 123-145, 2007.

47. Wallert M, Ziegler M, Wang X, Maluenda A, Xu X, Yap ML, Witt R, Giles C, Kluge S, Hortmann M, et al: $\alpha$-Tocopherol preserves cardiac function by reducing oxidative stress and inflammation in ischemia/reperfusion injury. Redox Biol 26: $101292,2019$.
48. Chen QM and Maltagliati AJ: Nrf2 at the heart of oxidative stress and cardiac protection. Physiol Genomics 50: 77-97, 2018.

49. Lv Y, Bing Q, Lv Z, Xue J, Li S, Han B, Yang Q, Wang X and Zhang Z: Imidacloprid-induced liver fibrosis in quails via activation of the TGF- $\beta 1 /$ Smad pathway. Sci Total Environ 705 , 135915,2020

50. Han B, Li S, Lv Y, Yang D, Li J, Yang Q, Wu P, Lv Z and Zhang Z: Dietary melatonin attenuates chromium-induced lung injury via activating the Sirt1/Pgc-1 $\alpha /$ Nrf2 pathway. Food Funct 10: 5555 $5565,2019$.

51. Jakobs P, Serbulea V, Leitinger N, Eckers A and Haendeler J: Nuclear factor (Erythroid-Derived 2)-like 2 and thioredoxin-1 in atherosclerosis and ischemia/reperfusion injury in the heart. Antioxid Redox Signal 26: 630-644, 2017.

52. Hun Lee J, Shu L, Fuentes F, Su ZY and Tony Kong AN: Cancer chemoprevention by traditional chinese herbal medicine and dietary phytochemicals: Targeting nrf2-mediated oxidative stress/anti-inflammatory responses, epigenetics, and cancer stem cells. J Tradit Complement Med 3: 69-79, 2013

53. Luo JF, Shen XY, Lio CK, Dai Y, Cheng CS, Liu JX, Yao YD, $\mathrm{Yu}$ Y, Xie Y, Luo P, et al: Activation of $\mathrm{Nrf} 2 / \mathrm{HO}-1$ pathway by nardochinoid $\mathrm{C}$ inhibits inflammation and oxidative stress in lipopolysaccharide-stimulated macrophages. Front Pharmacol 9: 911, 2018

54. Yang S, Chou G and Li Q: Cardioprotective role of azafrin in against myocardial injury in rats via activation of the Nrf2-ARE pathway. Phytomedicine 47: 12-22, 2018.

55. Chiu PY, Chen N, Leong PK, Leung HY and Ko KM: Schisandrin $\mathrm{B}$ elicits a glutathione antioxidant response and protects agains apoptosis via the redox-sensitive ERK/Nrf2 pathway in H9c2 cells. Mol Cell Biochem 350: 237-250, 2011.

56. Potenza MA, Sgarra L, Nacci C, Leo V, De Salvia MA and Montagnani M: Activation of AMPK/SIRT1 axis is required for adiponectin-mediated preconditioning on myocardial ischemia-reperfusion (I/R) injury in rats. PLoS One 14: e0210654, 2019.

57. Zhang Y, Wang Y, Xu J, Tian F, Hu S, Chen Y ans Fu Z: Melatonin attenuates myocardial ischemia-reperfusion injury via improving mitochondrial fusion/mitophagy and activating the AMPK-OPA1 signaling pathways. J Pineal Res 66: e12542, 2019

58. Duan J, Guan Y, Mu F, Guo C, Zhang E, Yin Y, Wei G, Zhu Y, Cui J, Cao J, et al: Protective effect of butin against ischemia/reperfusion-induced myocardial injury in diabetic mice: Involvement of the AMPK/GSK-3 $\beta / \mathrm{Nrf} 2$ signaling pathway. Sci Rep 7: 41491, 2017.

59. Tanaka M, Kishimoto Y, Sasaki M, Sato A, Kamiya T, Kondo K and Iida K: Terminalia bellirica (Gaertn.) Roxb. Extract and gallic acid attenuate LPS-induced inflammation and oxidative stress via MAPK/NF- $\kappa$ B and Akt/AMPK/Nrf2 pathways. Oxid Med Cell Longev 2018: 9364364, 2018.

60. Hou X, Fu M, Cheng B, Kang Y and Xie D: Galanthamine improves myocardial ischemia-reperfusion-induced cardiac dysfunction, endoplasmic reticulum stress-related apoptosis, and myocardial fibrosis by suppressing AMPK/Nrf2 pathway in rats. Ann Transl Med 7: 634, 2019.

61. Huang S, Li H and Ge J: A cardioprotective insight of the cystathionine $\gamma$-lyase/hydrogen sulfide pathway. Int J Cardiol Heart Vasc 7: 51-57, 2015.

62. Peake BF, Nicholson CK, Lambert JP, Hood RL, Amin H, Amin S and Calvert JW: Hydrogen sulfide preconditions the $\mathrm{db} / \mathrm{db}$ diabetic mouse heart against ischemia-reperfusion injury by activating Nrf2 signaling in an Erk-dependent manner. Am J Physiol Heart Circ Physiol 304: H1215-H224, 2013.

63. Fang XX, Wang H, Han D, Xie E, Yang X, Wei J, Gu S, Gao F, Zhu N, Yin X, et al: Ferroptosis as a target for protection against cardiomyopathy. Proc Natl Acad Sci USA 116: 2672-2680, 2019.

64. Dodson M, Castro-Portuguez R and Zhang DD: NRF2 plays a critical role in mitigating lipid peroxidation and ferroptosis. Redox Biol 23: 101107, 2019

This work is licensed under a Creative Commons Attribution-NonCommercial-NoDerivatives 4.0 International (CC BY-NC-ND 4.0) License. 